\title{
Labyrinthe
}

$7 \mid 2000$

Numéro 7

\section{Un faux révélé}

Analyse diplomatique d'une lettre autographe attribuée à Charles VIII

\section{Claude Jeay}

\section{(2) OpenEdition}

\section{Journals}

Édition électronique

URL : http://journals.openedition.org/labyrinthe/808

DOI : $10.4000 /$ labyrinthe.808

ISSN : 1950-6031

Éditeur

Hermann

\section{Édition imprimée}

Date de publication : 30 octobre 2000

Pagination : 145-151

\section{Référence électronique}

Claude Jeay, « Un faux révélé », Labyrinthe [En ligne], 7 | 2000, mis en ligne le 09 juillet 2005, consulté le 19 avril 2019. URL : http://journals.openedition.org/labyrinthe/808 ; DOI : 10.4000/labyrinthe.808

Ce document a été généré automatiquement le 19 avril 2019.

Propriété intellectuelle 


\section{Un faux révélé}

Analyse diplomatique d'une lettre autographe attribuée à Charles VIII

\section{Claude Jeay}

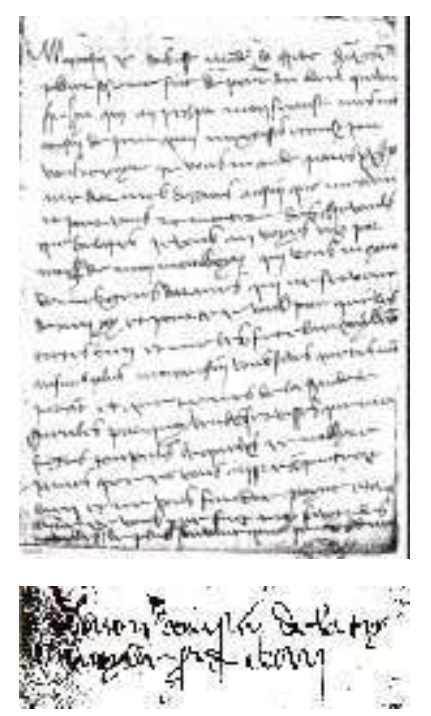

1 La critique des faux s'est progressivement affirmée comme une composante essentielle de la diplomatique. L'étude du document est l'objet d'enjeux nouveaux, notamment au plan culturel. En effet, outre l'apport historique évident (il peut être fondamental de savoir, pour la compréhension des faits, si un certain document est authentique), la critique des faux apporte un regard nouveau sur l'historiographie (la description du faux renseigne sur la vision qu'a le faussaire du passé). En voici un nouvel exemple.

2 Une lettre a longtemps été attribuée à Charles VIII lui-même, malgré quelques réserves émises notamment par les éditeurs de la correspondance de ce roi au début du siècle. Il s'agit d'une bande de papier allongée écrite sur les deux faces. Elle est présentée comme autographe dans l'ouvrage publié par Louis de La Trémoïlle ${ }^{1}$. Un peu à la manière d'une enquête policière, l'accumulation d'indices (notamment l'étude de la signature) permet de prouver définitivement que la lettre est fausse ${ }^{2}$.

Les signatures royales 
3 La signature de Charles VIII (1483-1498) est très sobre, dépourvue de paraphe. La lettre initiale $C$ est formée de deux ou trois éléments un peu maniérés, alors que la lettre $l$ est à peine ornée. Les caractères sont grands : le nom peut mesurer jusqu'à sept centimètres de long ${ }^{3}$. Leur dimension importante, sans précédent jusqu'alors pour une signature royale, compense dans une certaine mesure la mise en scène fastueuse qui était de mise dans la deuxième moitié $\mathrm{du} \mathrm{XIV}^{\mathrm{e}}$ siècle, associant le nom à un paraphe compliqué. Le nom du roi apparaît désormais magistralement, avec une certaine solennité.

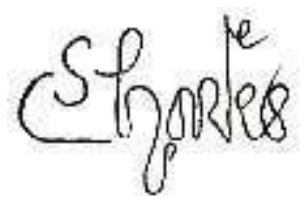

Fig. 1 - Reproduction.

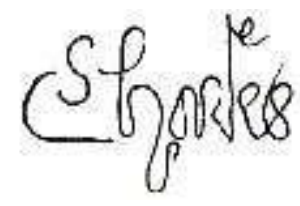

Fig. 2 - Reproduction.

Il est deux modèles distincts : le premier (fig. 1) est employé jusqu'au milieu de l'année 1495. Un second type (fig. 2) lui succède alors définitivement ${ }^{4}$. Il n'est pas facile de distinguer immédiatement entre les deux si l'on ne s'intéresse pas à la lettre l. Jusqu'à la fin du mois de juillet 1495, celle-ci forme une boucle fermée assez rigide. Par la suite, elle est constituée d'une verticale fragile qui se termine par une petite boucle ouverte s'étirant vers le haut. Plus difficile à remarquer, le $s$ final n'est pas toujours réalisé de la même manière ; il faut s'appliquer à en suivre le tracé pour s'en rendre compte ${ }^{5}$. C'est là un élément de datation précis qui permet de diviser le règne en deux parties inégales : la première va du début du règne jusqu'en juillet 1495, la seconde couvre les dernières années seulement (juillet 1495avril 1498). Sans être suffisant, ce critère permet une première estimation de la date ${ }^{6}$. Pour l'heure, ce changement reste inexpliqué. L'immense majorité des signatures de Charles VIII - qu'elles soient du premier ou du second type n'est sans doute pas de la main du roi.

Le problème de l'autographie et des signatures authentiques

5 L'édition de la correspondance de Charles VIII recense plus de mille lettres ${ }^{7}$. Elles n'ont pas le style des missives de Louis XI, dont on perçoit encore le souffle de la dictée. La correspondance de Charles VIII est au contraire froide et très peu personnelle ${ }^{8}$. C'est à se demander si le roi a fait plus que donner des instructions à ses secrétaires, tant il semble peu impliqué. Le problème de l'autographie se pose donc tout naturellement ${ }^{9}$.

Malgré des études effectuées sous le regard permanent de son père, Charles VIII est assez peu familier de l'écrit. Les signatures autographes sont peu nombreuses (fig. 3 et 4) ; elles se distinguent des autres par leur caractère malhabile, peut-être davantage dû aux tremblements de la main du roi qu'au manque de pratique de l'écriture. Zacharie Contarini, à la fin de la description sans concessions ${ }^{10}$ qu'il consacre au roi de France, ne peut s'empêcher de les faire remarquer : « le roi de France [...] est âgé de vingt-deux ans ${ }^{11}$, petit et mal fait de sa personne, laid de visage avec de gros yeux blancs beaucoup plus aptes à voir mal que bien, le nez aquilin également grand et gros plus qu'il ne convient; les lèvres aussi sont grosses et il les tient continuellement ouvertes; il a dans la main certains 
mouvements nerveux qui semblent fort laids à voir, et il est lent à s'exprimer ${ }^{12}$ ». Peu de signatures de Charles VIII ont un aspect maladroit ${ }^{13}$; le roi aurait donc peu signé de lettres, préférant, pour des raisons que l'on comprend, déléguer sa signature à un "secrétaire de la main $»^{14}$. Il est cependant probable que des signatures un peu moins malhabiles soient de la main de Charles VIII ${ }^{15}$. La signature régulière et maîtrisée apposée sur la lettre ne ressemble pas aux signatures tremblées qui sont incontestablement autographes. Elle rappelle en revanche celle d'un « secrétaire de la main ", telles qu'on en voit au bas des lettres et des mandements.

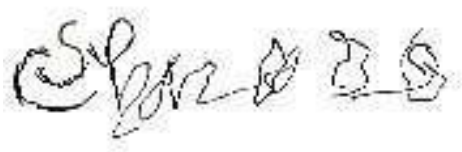

Fig. 3 - Arch. nat., X-fol. 180.

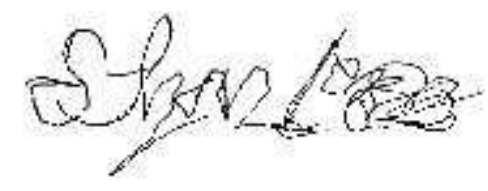

Fig. 4 - Arch. nat., X.fol. 190.

7 Le faussaire ignorait la différence entre les signatures autographes et les autres ; les premières sont rares et sans doute n'en avait-il jamais vues. Il a donc (parfaitement) imité une signature royale qu'il prenait pour telle alors qu'elle était apposée par un « secrétaire de la main ». Cette fausse signature, tracée au bas d'un document non autographe, aurait parfaitement fait illusion et son authenticité n'aurait jamais été contestée. Mais le faussaire a voulu aller trop loin et a accumulé les erreurs.

De nombreuses erreurs L'écriture

8 Pour l'heure, il semble que Charles VIII n'a jamais tracé que son nom ; on ne connaît aucune phrase de sa main, pas plus que le moindre mot. L'écriture de la lettre présente des faiblesses et des aberrations dans le tracé des lettres (groupes mal formés, forme des lettres $r, v, f, h, j$ et $g$ parfois erronée selon leur place dans le mot, présence étrange d'un accent aigu, cursivité injustifiée.... ${ }^{16}$. Bref, l'écriture employée ne ressemble que de loin à une écriture en usage à la fin du $\mathrm{Xv}^{\mathrm{e}}$ siècle ; elle tient plutôt d'un amalgame fantaisiste de signes empruntés aux $\mathrm{xv}^{\mathrm{e}}$ et $\mathrm{xVI}^{\mathrm{e}}$ siècles. Texte et signature, en opposant l'irrégularité et la maîtrise, créent un fort contraste ; l'ensemble est hétéroclite et l'apparence qui en résulte incohérente. Le faussaire ne possédait pas une bonne connaissance des écritures anciennes, hormis quelques notions confuses.

Une orthographe fantaisiste n'est pas (toujours) un signe d'autographie

L'orthographe et le vocabulaire sont aussi hasardeux que l'écriture. L'accumulation de bizarreries graphiques jusqu'à l'excès renforce la méfiance plutôt que le caractère autographe du document. En outre, le mot « remonte » (des chevaux, ligne 7) n'est attesté dans les dictionnaires qu'à partir des années $1670-1680^{17}$. Le contenu de cette lettre, loin de dissiper ces soupçons dévoile la raison d'être de ce faux : le panégyrique de Louis II de la Trémoïlle rédigé par le roi lui-même ! Il devient la plus belle pièce de l'échange épistolaire ; on comprend la fierté du descendant de cette illustre famille qui, en la publiant, a voulu s'approprier une parcelle de la gloire de son ancêtre.

La date 
10 Si le faussaire a commis des erreurs dans l'élaboration de cette lettre, il a cependant pris soin de vérifier la date. Toutes les lettres de l'ou-vrage concernent la guerre de Bretagne (1488) : le 16 avril 1488 tombait bien un mercredi. Mais l'indication de l'heure est doublement sujette à caution : elle n'est en principe jamais mentionnée (à quelques exceptions près) et elle est indiquée par un chiffre romain alors qu'elle devrait au moins être en toutes lettres ${ }^{18}$.

Conclusion : un faux tardif

11 La démarche du faussaire est désormais évidente. Il lui a fallu inventer l'écriture de toutes pièces, puisqu'il ne pouvait s'inspirer d'aucun original (peut-être s'est-il contenté de déformer jusqu'à l'aberration des écritures des notaires de la chancellerie). Pour la signature en revanche, il avait à sa disposition des dizaines de modèles. Mais c'était sans savoir que seules quelques-unes sont de la main du roi. Il a donc bien recopié une signature de Charles VIII, mais tracée par un "secrétaire de la main ». Au total, tous les éléments constitutifs de la lettre sont erronés. Le faussaire disposait à l'origine d'une série de missives proches dans le temps traitant toutes du même sujet ; le contexte rêvé pour insérer une fausse lettre dans la liasse, mais il n'a fait que prendre des initiatives malheureuses et faire les mauvais choix. La signature infirme finalement ce qu'elle devait prouver : l'autographie de la lettre. Le faussaire, piètre diplomatiste, est démasqué19.

Transcription

Mon cousin, je vous usse mandé la chete de vostre pillier se ne fut de peur du deul qu'aviés hehu qui anpechat mon servisse ; més, mon cousin, de peur qu'an megrissés et ausy pour vous rejouyr, je vous mande qu'arés XX home d'armes de crues ansin

que m'ecrivites. Et pour vous remonter des chevauls que brulates, je vous anvoyes ung par mons de Monmorrilloyn, qui vous maynne de mes gensdarmes qui ne servent de rien ycy; et pour ce je vous prie que les tretés bien et me les fete bien

vayllans. Au surplus, mon cousin, vous savés qu'etes mon parant et que tenés de la bande de gueules; par quoy veu les servisses que me fetes tout-jours, lequelxs je n'oblyré jamés, que je vous an recompanceré bien et ne vous faudré poynt. Et ancoure je vous prie fet moy savoyr des novelles le plus souvant que pourés. Adieu mon cousin, que je

prie a Dieu qui vous doynt vous desirs tous accomplis.

Escrit au Plesys du Parc ce mercredy xvi jour d'avril a j hure après mydy. 


\section{NOTES}

1. Louis de La Trémoïlle, Correspondance de Charles VIII et de ses conseillers avec Louis II de la Trémoille pendant la guerre de Bretagne (1488), Paris, 1875, p. 48 et planche 45.

2. Nous remercions Olivier Guyotjeannin et Marc Smith, professeurs à l'École des chartes, de leurs remarques et conseils.

3. Bibl. nat. de Fr., fr. 25716, n 95 (vol. des « Chartes royales »). À mesure que l'on avance dans le règne, la signature prend de l'ampleur, comme si elle cherchait à combler l'espace inutilisé de plus en plus vaste, au bas des documents. À partir de 1489-1490, la signature de Charles VIII mesure très souvent plus de 6 centimètres de long. Voir dans le même volume les $\mathrm{n}^{\circ \mathrm{s}} 87,88$, 90. Et Bibl. nat. de Fr., fr. 25717, n 106 et 135 par exemple.

4. R. Scheurer, «La chancellerie de France et les écritures royales au temps de Charles VII et Louis XI », dans Positions des thèses de l'École des chartes, 1962, p. 111.

5. Il faut se fonder sur la modification de la lettre $l$. En effet, il est des cas ou le $s$ final est tracé de la première manière ; c'est donc un critère supplémentaire, mais qui ne doit pas induire en erreur.

6. Le millésime n'est pas indiqué sur les lettres hors chancellerie, d'où l'importance de tout élément permettant de les situer dans le temps.

7. Ce qui est très peu. Celle de son père Louis XI en compte plus de 2200, en dépit de toutes celles que l'on sait perdues - pour un règne plus long il est vrai.

8. Lettres de Charles VIII, roi de France, publiées d'après les originaux pour la Société de l'histoire de France, par P. Pélicier et B. de Mandrot, Paris, 1898-1905, p. I : « La lecture de [la] correspondance [de Charles VIII], rarement animée par un éclair d'originalité, ne permet pas une bien favorable opinion ». Et plus loin : « En feuilletant sa correspondance, on n'arrive pas à le prendre sur le fait, à vivre avec lui, à se représenter tout entier, comme lorsqu'on lit les missives d'un Louis XI ».

9. R. Scheurer, art. cit., p. 111 : « Le roi [Charles VIII] ne signait pas toujours de sa main ». Les signatures autographes sont très rares, voir infra.

10. Voir par exemple J. Ehrmann, « Un portrait inédit de Charles VIII par Jean Bourdichon ", Bulletin de la Société nationale des Antiquaires de France, 1978-1979, p. 265-272. L'article donne des éléments bibliographiques sur les portraits de ce roi.

11. L'entrevue a lieu en 1492.

12. H.-F. Delaborde, L'Expédition de Charles VIII en Italie : histoire diplomatique et militaire, Paris, 1888, p. 224.

13. Arch. nat., X", 9321, fol. 180 et fol. 190. Et Bibl. nat. de Fr., fr. 6602, fol. 150 et fol. 159. Les signatures sont très allongées, beaucoup plus que celles tracées régulièrement.

14. Depuis la fin du Moyen Âge, le roi s'entoure de quelques secrétaires dignes de confiance qu'il autorise à signer de son nom. Mais l'expression de secrétaire de la main est plus tardive, c'est pourquoi elle est ici employée avec des guillemets.

15. Peut-être Charles VIII connaissait-il ces problèmes de tremblement depuis l'enfance confiance à qui il donne l'autorisation de signer de son nom. Cela expliquerait (au moins partiellement) le système de reproduction par xylographie - à l'aide d'un tampon - de la signature royale, utilisé par les Beaujeu pendant la régence. Ce procédé de reproduction est dénoncé par Louis d'Orléans en janvier 1485, comme une source d'abus. Il est alors en 
effet possible de soustraire nombre de documents à l'avis du jeune roi. Aucune signature en manière de tampon n'a été retrouvée au cours des dépouillements. Voir aussi J.

Liniger, Philippe de Commynes, Paris, 1978, p. 255.

16. Nous remercions M. Smith d'avoir accepté d'étudier cette lettre et d'en livrer son analyse dont certaines conclusions sont reprises ici.

17. Nous remercions $O$. Guyotjeannin de cette précision.

18. On attend « une ». Le faussaire emploie la lettre $i$ au lieu de la lettre $j$; le dernier jambage - ou l'unique, comme dans le cas présent - est toujours plus long.

19. Ce faux pourrait dater de la première moitié du XIX ${ }^{e}$ siècle. La description du faux renseigne sur la vision qu'avait le faussaire du règne de Charles VIII, et plus généralement du Moyen Âge finissant. Il suffit de dire que ces temps lui semblent bien obscurs et éloignés.

\section{AUTEUR}

\section{CLAUDE JEAY}

Claude JEAY, après un DEA à l'EPHE sous la direction de Michel Pastoureau (Des signatures et des rois, 1999), a soutenu une thèse à l'École des chartes en mars 2000 (Du sceau à la signature) sous la direction de Olivier Guyotjeannin et Michel Pastoureau. Actuellement conservateur stagiaire à l'École Nationale du Patrimoine, il prépare un doctorat à l'EPHE sous la direction de Michel Pastoureau. c-jeay(at)voila.fr 